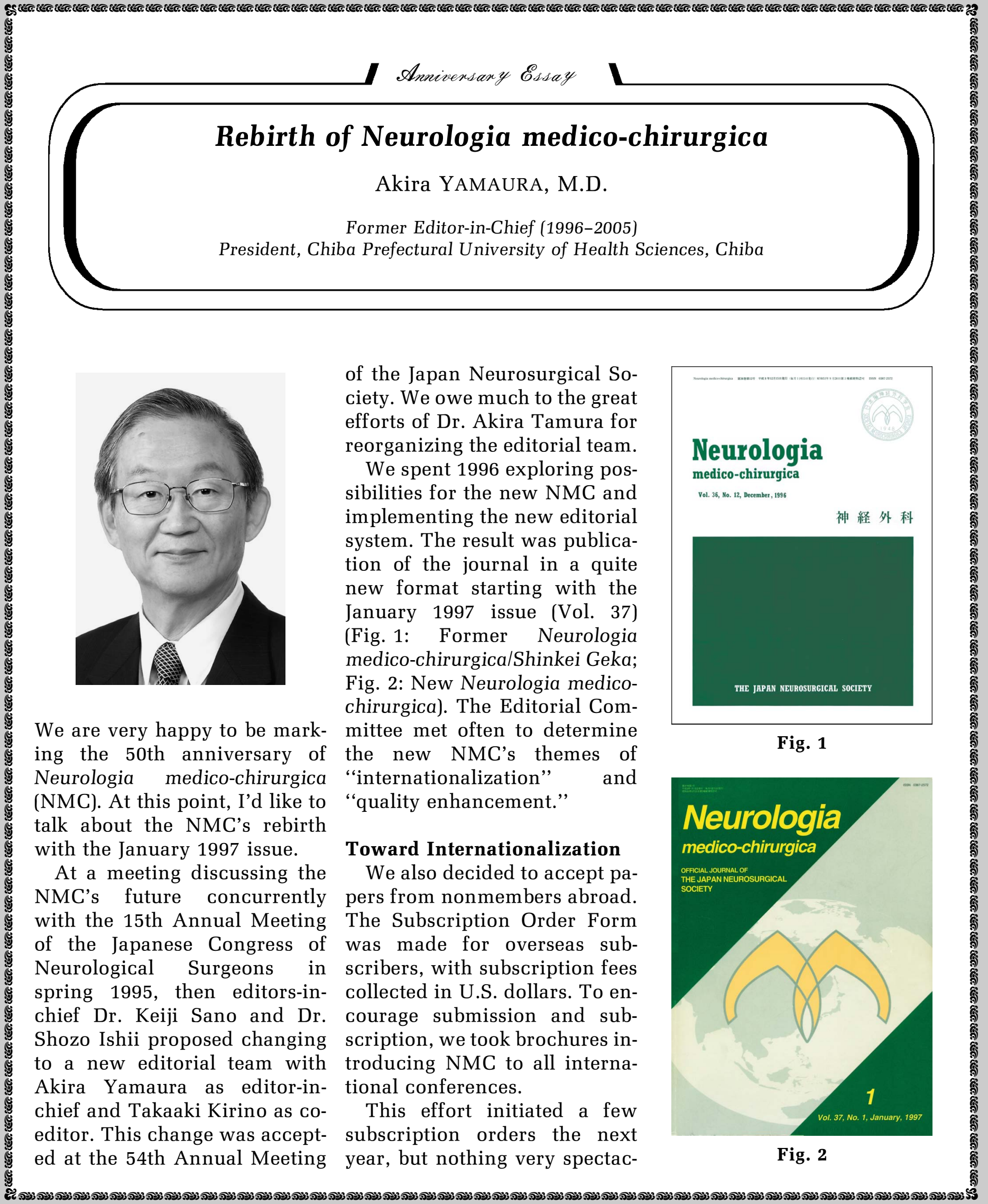


ular. The Editorial Committee decided to prioritize increasing the number of citations of papers in NMC, rather than increasing revenue through subscriptions.

The International Advisory Board's 44 members were chosen from the United States, European countries, Brazil, Taiwan, and China to cover each field. We also added comments to each paper, since comments by international experts greatly encouraged authors and readers.

The NMC's cover was dramatically redesigned to incorporate the NMC's original green at the upper left and lower right resembling a green curtain opening upon the Earth seen from outer space. This rotates 360 degrees, starting with Japan in the center of the January cover, then gradually disappearing and reappearing as the world turns. We adopted the A4 size to match global trends, maintaining paper quality while improving readability.

Toward Quality Enhancement We entirely revised the "In- structions for Authors" to emphasize research/clinical ethics, and added "Rapid Communications" for the newest information originating in Japan and "Special Topics" addressing social changes. "Essay Club" was added as a "coffee break." The first essay was written by Dr. Keiji Sano on "Haiku." Unfortunately, only a few essays have been contributed.

The review process is essential for enhancing quality. Taking into consideration the need for review promptness and expertise, 49 members from the Review Board, 28 members from the Editorial Board, and 6 editorial staff were available.

Taking into account the specialties in review, the editor-inchief chose and designated an Editorial Board member and two Review Board members to review all papers. Each member carefully reviews and provides advice to enhance the quality of papers, always demonstrating passion and dedication to their profession.

Impact factor is an international evaluation of journals.
Our "first" impact factor rating was 0.387 in 2000 ! The impact factor of NMC has gradually increased, reaching 0.473 in 2007. However, we realized that it was difficult to raise impact factor in a specialized field such as neurosurgery where major American and European journals dominate.

\section{And Also -}

With an increasing number of submissions from Asian nations, suggestions have been made to change the journal title to, for example, the "Asian Journal of Neurosurgery." We are now broaching this subject among members for feedback.

In compliance with the agreement made at the first meeting of the Editorial Committee (February 3, 1996), the former editor-in-chief and co-editor withdrew from the editing task in August 2005 as they retired from their university posts, leaving the future development of the Journal to Dr. Yoichi Katayama and others. I thank Dr. Takaaki Kirino for his many valuable suggestions and ongoing efforts as co-editor. 